\title{
La Educación en México como un Sistema Complejo
}

\author{
Hilda Beatriz Ramirez Moreno \\ Profesor Investigador de la Facultad de Contaduria y \\ Administracion de \\ la Universidad Autónoma de Baja California, México \\ ramirezmb@uabc.edu.mx \\ 0000-0003-4816-8382 \\ Nora Osuna-Millán \\ Profesor Investigador de la Facultad de Contaduria y \\ Administracion de \\ la Universidad Autónoma de Baja California, México \\ nora.osuna@uabc.edu.mx \\ 0000-0001-5719-7682 \\ Jesús Manuel Niebla Zatarain \\ Profesor Investigador de la Facultad de Derecho, \\ en la Universidad Autónoma de Sinaloa, México \\ j.niebla@uas.edu.mx
}

Recepción: 24/09/2021

Aceptación:29/10/2021

\section{Resumen}

El presente trabajo permite visualizar la obligatoriedad de la educación básica y media superior, la manera en que está organizado el sistema educativo en México, así también se destacan los principales actores que participan en los procesos de enseñanza aprendizaje, las diversidad en las relaciones que determinan a la Educación como un sistema complejo, por lo que es necesario atender o aplicar los principios básicos de la complejidad, lo cual permitirá transformar el proceso Educativo, por medio del pensamiento sistémico, basado en la relación de los elementos principales, atendiendo la comunicación y retroalimentación, todo ello basados en la conciencia, adaptación, autoorganización, de los involucrados entre ellos las instituciones, profesores, alumnos, organizaciones privadas y sin fines de lucro, la secretaría de educación pública, el poder legislativo representado por la cámara de diputados los cuales actúan como gestores de recursos para establecer el mejor clima para desarrollar un Sistema Educativo de calidad que responda a las necesidades del entorno.

Palabras clave: Educación, sistema complejo, conciencia,

\section{Abstract}

This work allows to visualize the compulsory nature of basic and upper secondary education, the way in which the educational system in Mexico is organized, as well as the main actors that participate in the teaching-learning processes, the diversity in the relationships that determine to Education as a complex system, so it is necessary to 
attend or apply the basic principles of complexity, which will allow to transform the Educational process, through systemic thinking, based on the relationship of the main elements, attending to communication and feedback, all based on the awareness, adaptation, self-organization, of those involved, including institutions, teachers, students, private and non-profit organizations, the public education secretariat, the legislative branch represented by the chamber of deputies which act as resource managers to establish the best climate for development roll out a quality Educational System that responds to the needs of the environment.

Keywords: Education, complex system, conscience.

\section{Introducción}

\subsection{Educación Obligatoria en México}

La Constitución Política de los Estados Unidos Mexicanos (CPEUM) en su artículo 30 indica el derecho de todos a la educación. donde los 31 estados, Ciudad de México y Municipios- deben dictar y proveer la educación impartirán y garantizarán la educación inicial, preescolar, primaria, secundaria, media superior y superior (todos los niveles). La educación educación básica y la media superior serán obligatorias, la educación superior se promoverán los medios de acceso por las instituciones públicas, Cámara de diputados. (2021).

La figura 1 nos muestra cómo se clasifican los niveles educativos en México, para proporcionar el servicio a la sociedad y dar cumplimiento a la Constitución Política de los Estados Unidos Mexicanos en su artículo 3ro.

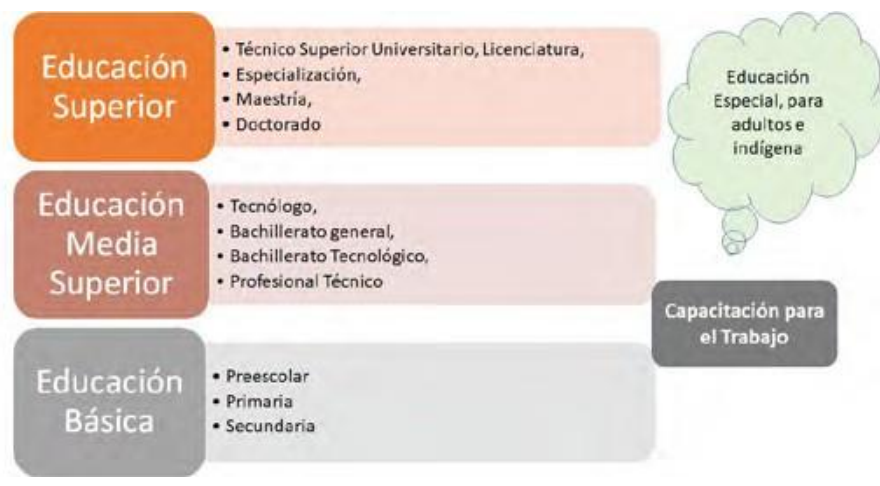

Figura 1. Estructura de la Educación en México

La Educación en México, según la Secretaría de Educación Pública (2019) debe considerar la Educación especial, la Educación Indigena ya que una de las características más sobresalientes de nuestro país es la diversidad cultural la cual está compuesta por diversas etnias por lo tanto es indispensable su consideración, la Educación para adultos mayores promueve la adqui- sición de competencias académicas básicas las cuales certificarán su experiencia informal y permitirán una mejor inserción en el mercado laboral.

\section{Sistema Educativo Nacional}

Se conoce al Sistema Educativo Nacional según el artículo 31 de la Ley General de Educación, Cámara de Diputados (2019) como el conjunto de grupos de interés, instituciones y procesos que están relacionados en la tarea proporcionar y recibir el servicio relacionado con la educación, a la que está obligada el gobierno, donde se incluye desde la educación Primaria hasta la Educación superior. Aquí se involucran estructuras, Instituciones, profesores, relaciones, familias, sociedad mexicana en general; los cuales forman parte del todo que permite lograr los objetivos que cada nivel educativo persigue.

El Sistema Educativo funciona por medio de la interacción entre los principales elementos que lo componen, los impulsores que se dan entre ellos y el entorno permite que la adaptación a situaciones que emergen se atenúen, ver la Figura 2.

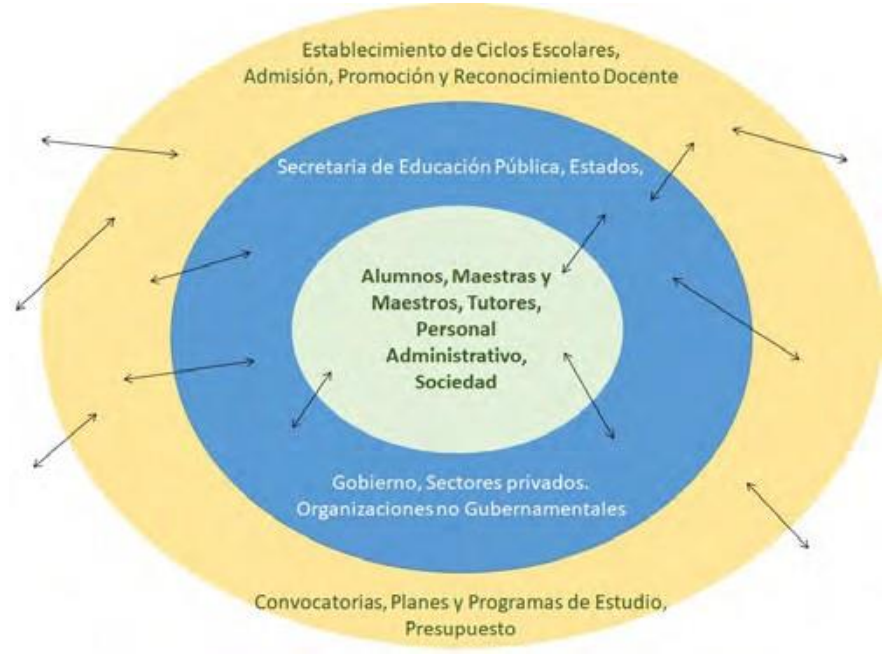

Figura 2. Sistema Educativo Nacional en México

\subsection{Educación y complejidad}

La educación se considera un sistema complejo por el número de relaciones que los actores participantes generan; al considerarse esta como un sistema complejo permitirá crear escenarios cercanos a la realidad del estudiante y del docente, lo que permitirá estrategias que proporcionen mejoras en este sector, Serghie (2015).

Los sistemas complejos principalmente se caracterizan por desarrollar dinámicas irrepetibles, sin un periodo establecido, con apariciones repentinas, además de otras características, y según Maldonado, C. E. ( 2014) estos son los rasgos básicos de la complejidad. 
Los sistemas complejos pueden distinguirse por el comportamiento de los actores y las relaciones entre ellos, el cual resulta ser de manera dinámica, por medio de la emergencia de actuaciones dispersas.

\section{Perspectiva de aspecto sistémico de la compleji- dad \\ Sistemas}

Para entender que es un sistema complejo nos remontamos a los inicios de la Teoría General de Sistemas (TGS) donde surge gracias a los trabajos del alemán Ludwig Von Bertalanffy (Goncalves, 2011) que se percató de la necesidad de conceptos unificadores en las ciencias sociales y naturales. La teoría general de sistemas (TGS) se aplica a todas las ciencias y ha servido de base para la integración del conocimiento científico, esta teoría busca las propiedades en común de sus componentes así como la integración de todas sus partes para lograr su objetivo. La TGS se fundamenta en 3 premisas básicas (Hurtado, 2008):

1. Los sistemas existen dentro de sistemas

2. Los sistemas son abiertos.

3. Las funciones de un sistema dependen de su estructura.

Hay una gran variedad de conceptos de sistemas pero todas tienen en común sus elementos o partes en interacción para lograr un objetivo o meta o como lo define Vasquez, 2012, donde define que un sistema está conformado por un conjunto de objetos o componentes que interactúan entre sí para el logro de objetivos bajo un entorno.

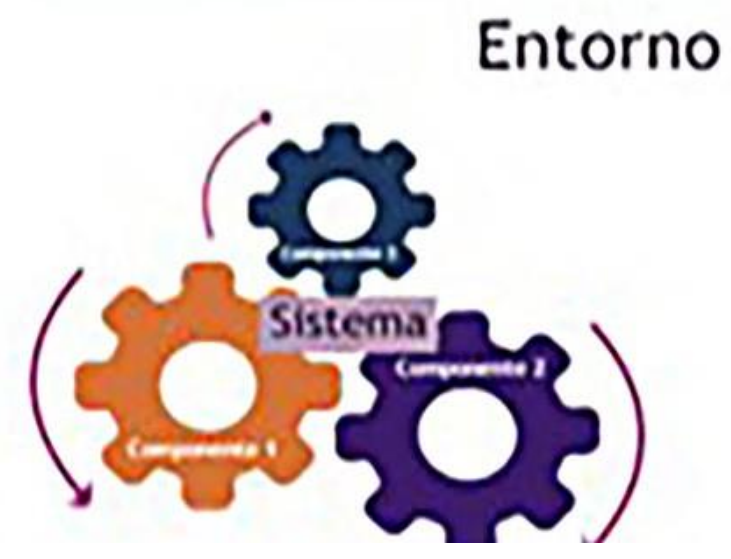

Figura 3. Concepto de sistema

En la actualidad la palabra sistema se utiliza en todas las ciencias porque implica unión, integridad, totalidad y partes en relación para lograr un mejor funcionamiento que de manera individual no lo podría alcanzar, algunos ejemplos de la diversidad de sistemas son: sistema fotográfico, sistema solar, sistema educativo, sistema de transporte, sistema judicial, como podemos observar la gran variedad de sistemas en la actualidad es muy diversa. Un sistema tiene la capacidad de que toda acción que produce influye en las otras partes y esto afecta el logro de su meta así como el entorno que rodea al sistema surgiendo el concepto de Sistema Complejo.

\section{Sistema Complejo}

El estudiar un sistema complejo va más a fondo que la integración de sus componentes generando una sinergia para lograr un mejor desarrollo y funcionamiento (EPG, 2021), como se muestra en la figura 4. Para entender el comportamiento del sistema complejo debemos de partir que sus componentes actúan de manera individual pero sus acciones influyen en los demás componentes y en el desarrollo del sistema para adaptarse y cumplir con su objetivo. Algunos ejemplos de sistemas complejos son las hormigas, el cerebro, la conciencia.

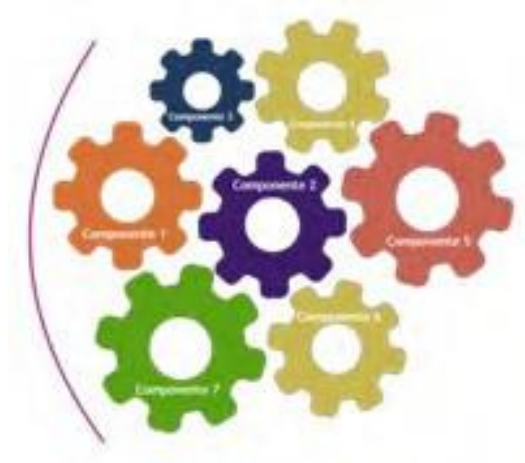

Figura42. Sistema Complejo

La conciencia es un sistema complejo, todos sabemos dónde está el cerebro pero donde se encuentra la conciencia, que órgano es, que forma tiene, cómo se fundamenta, esas son las preguntas por contestar.

\section{Educar la Conciencia}

Uno de los sistemas complejos con mayor inquietud es la conciencia a través de la educación. Gran parte de nuestro desarrollo lo realizamos en nuestra vida estudiantil, en los primeros años en la educación básica o hasta llegar a la educación superior o algún posgrado. Todo este tiempo contribuye al desarrollo de nuestra conciencia que es solo un elemento o componente de todo lo que integra la conciencia. Pero qué es la conciencia? La conciencia es la capacidad propia de los seres humanos de reconocerse a sí mismos, de tener conocimiento y percepción de su propia existencia y de su entorno. La conciencia está relacionada con la acti- 
vidad mental que implica un dominio por parte del propio individuo sobre sus sentidos (Significados 2021). La conciencia permite reunir lo vivido, lo que está pasando y lo que deseas entre otras cosas. Por ejemplo el respirar lo hacemos desde que nacemos, y no pensamos en estar respirando, pero nos hacemos conscientes cuando un cantante, un deportista o nosotros practicamos yoga utilizamos la respiración controlada, diafragmática o simplemente el control de cómo y cuándo respirar somos ya conscientes.

En el ámbito educativo la conciencia no regula el aprendizaje, la forma en la que usamos el proceso de enseñanza-aprendizaje, sino que la conciencia convierte lo aprendido en una narrativa que se usa en el concepto social de manera que en pocas palabras platicamos y compartimos lo que sabemos sin necesariamente estar enseñando (Davis, 2004).

Los enfoques de la conciencia (Capra, 2002; Davis, Sumara y Luce-Kapler, 2000; Thompson, 2001; Varela, Thompson y Rosch, 1991) representan una comprensión neurofenomenología de la conciencia, expresan como las experiencia de lectura literaria influye en el desarrollo del yo y del yo comprensión.

1) La conciencia es un proceso: La conciencia surge como resultado de complejas interacciones neurofisiológicas que ocurre en el cerebro al mismo tiempo que reconoce que no se pueden explicar, es simplemente un producto de esas interacciones (Edelman, 1992, 2004; Damasio, 1994, 1999; Stewart y Cohen, 1997; Capra, 2002).

2) La conciencia está en todas partes y en ninguna parte: La conciencia se experimenta como unificada y coherente, es un proceso global que involucra las actividades interpenetrantes de poblaciones neuronales distribuidas por todo el cerebro (Edelman, 2004).

3) La conciencia es un fenómeno emergente: Conciencia no surge del funcionamiento independiente y aislado de los individuos, sino que es un proceso elaborado que evoluciona a medida que el yo consciente y el "Otros" se entrelazan y se despliegan en una compleja coreografía de co-determinación.

\section{Conciencia, lectura y educación}

La lectura ofrece el desarrollo de la autoconciencia de sus propias mentes, los lectores necesitan tomar conciencia de sus propias prácticas de lectura de mentes, cuando toman conciencia de los personajes.

Educación y Lecturas

- Utilizar lecturas profundas en lugar de lecturas superficiales en el salón de clases, los estudiantes pueden utilizar estas lecturas para cuestio- nar sus propias creencias.

- Utilizar diferentes géneros como el hipertexto apoyan el desarrollo de la autoconciencia.

- Los lectores necesitan conectar con el texto con sus propios recuerdos e imaginaciones, al hacerlo, crean nuevos niveles de autoconciencia.

- El acceso a grandes cantidades de información no garantiza que la comprensión ocurra.

\section{Complejidad y proceso de Aprendizaje}

Estrada G. A. (2020) presenta siete principios que pretenden establecer una línea base de consenso de diversas teorías, tendencias, enfoques; al mismo tiempo que permita generar un aprendizaje más sólido en los estudiantes en cuanto a: conocimiento habilidades, aptitudes, valores, etc lo cual propiciará un mejor desarrollo e inserción en la vida profesional y personal del estudiante.

El primer principio es el sistémico que nos permite tratar un todo que funciona a través de la interrelación y la comunicación de las partes que lo componen, sin tratar las partes de manera aislada, por lo que el proceso de enseñanza no es solo responsabilidad del profesor y el estudiante, sino de todo el sistema educativo, donde una parte gestiona los recursos, otra se actualiza y genera el ambiente, estrategias para que la adquisición de conocimiento perdure y otros actores se aseguran de acumular y procesar el conocimiento.

El principio hologramático nos habla de la manera requiere estimular una forma diferente de concebir o visualizar la realidad, por lo que es necesario el análisis de diversos escenarios educativos, los cuales permitan revisar sus interacciones, lo cual da lugar a un sistema complejo.

Principio de retroalimentación, aqui Morin (1994, p. 99) destaca que la causa actúa sobre el efecto y el efecto sobre la causa, estableciendo así un ciclo de relación.

El Principio recursivo permite una relación de causa y efecto, donde puede orientar al sistema educativo para que sus actores generen un efecto de mejora continua que permita cambios en los métodos de enseñanza y genere un plan de estudios que atienda las competencias diversas de los futuros profesionistas.

Principio de autonomía/dependencia, el cual muestra la manera en que el ser humano lucha por ser independiente, pero no corta sus relaciones con la sociedad, así, el docente intenta generar un conocimiento técnico o disciplinario del tema que lo ocupa, pero no puede dejar de largo las habilidades interpersonales (soft skills) que deben acompañar el proceso de aprendizaje

Principio dialógico, este principio es por medio del cual las partes establecen diálogo con el todo, así destaca su oposición a la abstracción y la disyunción , las cuales favorecen el aislamiento de los objetos de estu- 
dio y las relaciones del entorno. Por lo que este principio favorece la relación entre los actores del sistema educativo, así como su transformación y evolución.

Principio del que conoce en todo conocimiento, Este principio trata sobre que el conocimiento es una reconstrucción /traducción en un periodo establecido, de acuerdo a una cultura, idioma o lenguaje. el cual realiza una persona. En el proceso de enseñanza, podemos deducir que de acuerdo a cada grupo específico, generación, región, etc. por lo que las técnicas y modelos de aprendizaje deberían de ser diferentes, esto podría lograrse por medio de la introducción y combinación de diversas disciplinas, lo cual enriquecería la educación al acumular diferentes perspectivas.

\section{Conclusión}

La Educación es un derecho constitucional que permite el crecimiento económico de la región, pero en la actualidad se enfrenta a los cambios inherentes de la evolución. El sistema educativo es el resultado de la interacción de múltiples elementos que generan una sinergia para lograr un mejor funcionamiento y así cumplir con sus objetivos. La complejidad en la educación se presenta a partir que sus elementos actúan de manera individual como:

estudiantes, maestros, personal y procesos administrativos, infraestructura, tecnologías de información, procesos de enseñanza aprendizaje, y políticas públicas por mencionar algunas pero sus acciones afectan a los demás componentes del sistema. Por lo tanto las instituciones educativas, se deben involucrar a los cambios de la evolución con las directrices internacionales hacia modelos innovadores de calidad y excelencia.

La complejidad se da en la conciencia, al surgir de las relaciones entre la memoria, imaginación y percepción, como un todo donde la educación puede nutrir en el contexto del aula. Al tratarse al proceso de enseñanza aprendizaje como un sistema complejo es imprescindible que los actores de este sistema respondan a los efectos que se reciben del entorno.

Las situaciones emergentes derivadas de la globalización, problemas como la pandemia originada por el covid-19, el avance de las Tecnologías de la información, exigen una mejor atención del proceso educativo y de investigación que generen soluciones más eficientes y sólidas. La complejidad permite generar soluciones por medio de la proyección y predicción de soluciones basados en simulaciones de escenarios.

\section{Referencias}

Cámara de Diputados. (2019). Ley General de Educación. 14 octubre 2021, de Diario Oficial de la Federacion Sitio web: http:// www.diputados.gob.mx/LeyesBiblio/pdf/LGE_300919.pdf
Camara de diputados. (2021). CONSTITUCIÓN POLÍTICA DE LOS ESTADOS UNIDOS MEXICANOS. 8 Octubre 2021, de Congreso de la Union Sitio web: http://www.diputados.gob.mx/LeyesBiblio/pdf/1_280521.pdf

Capra, F. (2002) The Hidden Connections: Integrating the Biological, Cognitive, and Social Dimensions of Life into a Science of Sustainability (New York, Doubleday)

Davis, B. (2004) Inventions of Teaching: A genealogy (Mahwah, $\mathrm{NJ}$, Lawrence Erlbaum Associates)

Damasio, A. (1994) Descartes' Error: Emotion, reason and the human brain (New York, G.P. Putnam's Sons).

Damasio, A. (1999) The Feeling of What Happens: Body and emotion in the making of consciousness (San Diego, New York, and London, Harcourt Inc.).

Estrada, G. A.. (2020). Los principios de la complejidad y su aporte al proceso de enseñanza. 20 octubre 2021, de Scielo Brasil Sitio web: https://www.scielo.br/j/ensaio/a/b4CvmDH3fNCRvZT3K3MrQnj/?lang=es

Edelman, G. (2004) Wider than the Sky: The Phenomenal Gift of Consciousness (New Haven, Yale University Press).

EPG (2021). Blog Escuela de Posgrado, Universidad Continental. ¿Qué es un sistema complejo y cómo funciona en el sector público?. Se consultó el 4 nov. 2021. https://blogposgrado. ucontinental.edu.pe/que-es-un-sistema-complejo-y-comofunciona-en-el-sector-publico

Goncalves, M. (21 de 12 de 2011). http://teoriadelossistemasmaryg.blogspot.mx. Obtenido de EL ENFOQUE DE SISTEMAS: http://teoriadelossistemasmaryg.blogspot.mx/2011/10/ elenfoque-de-sistemas-sistemas.html

Hurtado, D. C. (2008). La teoría de sistemas se fundamenta en tres premisas básicas. En D. C. Hurtado, Principios de administración (pág. 100). Medellin: Fondo editorial ITM. Obtenido de Teoría de Sistemas: http://www.monografias.com/trabajos11/teosis/teosis.shtml

Maldonado, C.E. ( 2014). ¿QUÉ ES UN SISTEMA COMPLEJO?. Revista Colombiana de Filosofía de la Ciencia, 29, 71-93.

Secretaría de Educación Pública. (2018). La estructura del sistema educativo mexicano . 10 octubre 2021, de SITEAL Sitio web: https://siteal.iiep.unesco.org/sites/default/files/sit_accion_files/siteal_mexico_0101.pdf

Significados Significados.com. Disponible en: https://www.significados.com/conciencia/

Serghie, D.. (2018). Context in collaborative structures: transdisciplinarity. Network Intelligence Studies, 1, 5-12.

Stewart, I. \& Cohen, J. (1997) Figments of Reality: The evolution of the curious mind (Cambridge,Cambridge University Press). Thompson, E. (2001) Editor's Introduction: Empathy and consciousness. Journal of Consciousness Studies, 8, pp. 5-7.

Varela, F., Thompson, E. \& Rosch, E. (1991) The Embodied Mind (Cambridge MA, MIT Press).

Vasquez, R. G. (2012). Modelación del Desarrollo Sustentable en la ciudad de. En R. G. Vasquez, Desarrollo Sustentable en la ciudad de Piura (págs. 33-34). 